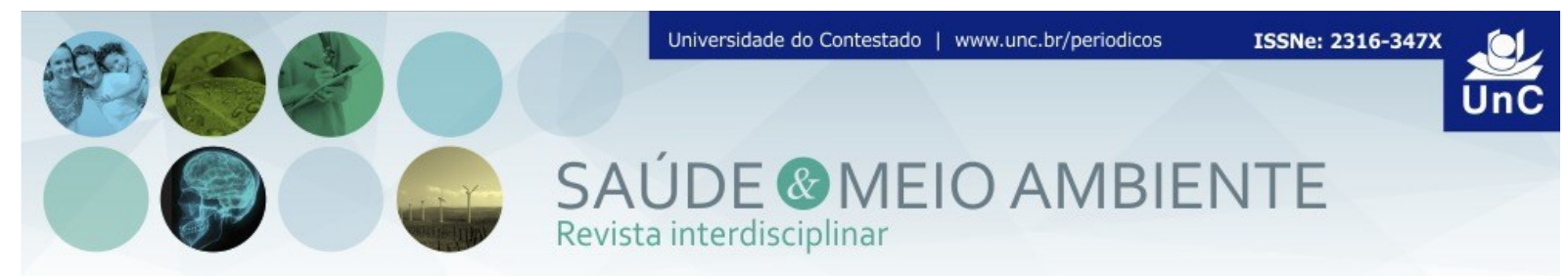

\title{
AVALIAÇÃO DE RUÍDO AMBIENTAL DA REGIÃO CENTRAL DA CIDADE DE UBERABA - MG
}

\section{ENVIRONMENTAL NOISE AVALIATION OF THE CENTRAL REGION UBERABA CITY - MG}

\author{
Michele Cristina Rufino Barbosa ${ }^{1}$ \\ Guilherme Wolf Gothelf ${ }^{2}$ \\ Lucas Araújo Carvalho ${ }^{3}$ \\ Mateus Scandar Prata ${ }^{4}$
}

\begin{abstract}
RESUMO
A poluição sonora ocorre quando um som ultrapassa o limite auditivo normal. Segundo a Organização Mundial de Saúde (OMS) a poluição sonora é a segunda maior causa de poluição, sendo considerada um problema de saúde pública mundial, pois afeta a saúde física e mental da população. É uma das fontes de poluição que mais geram incômodos atualmente em decorrência dos excessivos ruídos gerados por diferentes fontes. Devido aos diversos problemas de saúde acarretados pela poluição sonora o objetivo do estudo foi avaliar alguns pontos da região central de Uberaba - MG a fim de quantificar os níveis de intensidade sonora existentes e verificar se os níveis de ruído encontram-se acima do permitido causando efeitos negativos nos seres humanos. Foram realizadas medições de nível de pressão sonora de acordo com a NBR 10.151/2019 em 5 zonas da região central de Uberaba e em cada zona foram realizados 4 pontos de medição. Para cada ponto, a medição foi realizada de 1 em 1 minuto, totalizando 10 minutos. A partir dos valores de níveis de pressão sonora foi obtido o nível de pressão sonora equivalente que corresponde à média do ruído de cada local e este valor foi comparado com as normas referentes a poluição sonora. Os resultados obtidos ficaram acima dos estabelecidos por normas podendo acarretar danos à saúde da população que está submetida a esta poluição. Desta forma é necessário um melhor planejamento urbano da cidade e a incorporação de medidas de controle e de atenuação sonora para o bem-estar da comunidade.
\end{abstract}

Palavras-chave: Ruídos. Poluição sonora. Danos à saúde.

\footnotetext{
1Doutora em Geotecnia. Mestre em Engenharia Mineral. Engenheira Ambiental. Professora do Departamento de Engenharia Ambiental da Universidade Federal do Triângulo Mineiro (UFTM). Uberaba, Minas Gerais. Brasil. E-mail: michele.barbosa@uftm.edu.br

${ }^{2}$ Graduado em Engenharia Ambiental. Universidade Federal do Triângulo Mineiro (UFTM). Uberaba. Minas Gerais. Brasil. E-mail: guilhermewgothelf@gmail.com

${ }^{3}$ Graduado em Engenharia Civil. Universidade Federal do Triângulo Mineiro (UFTM). Uberaba. Minas Gerais. Brasil. E-mail: lucas.araujo 96@hotmail.com

${ }^{4}$ Mestre em Marketing. Graduado em Administração e Comunicação Social. Graduando do curso de Engenharia Ambiental da Universidade Federal do Triângulo Mineiro (UFTM). Uberaba - Minas Gerais. Brasil. E-mail: mateusscandarprata@hotmail.com
} 


\begin{abstract}
Noise pollution occur a sound exceeds the normal hearing limit. The World Health Organization (WHO) said that noise pollution is the second largest pollution cause, being considered a worldwide public health problem, as it affects the population physical and mental health. Today, it is one polltution sources that more affect the health human due to excessive noise generated by different sources. Because to the health problems noise pollution, the study objective was to evaluate some points in the Uberaba - MG central region to quantify the existing sound intensity levels and verify if the noise levels are above the noise levels permitted, cause impacts humans negative effects. Sound pressure level measurements were performed according to NBR 10.151 / 2019 in 5 zones Uberaba central region and for each zone 4 points measurement were made. The measurement was performed every 1 minute, totaling 10 minutes. The sound pressure level values, the equivalent sound pressure level was obtained, which corresponds to the average noise of each location and this value was compared with the noise pollution standards. The results obtained were above those established by standards and may cause damage to the population health that is subjected to this pollution. Thus, better city urban planning city and the incorporation of control measures and sound attenuation are necessary for the community wellbeing.
\end{abstract}

Key words: Noise. Noise Pollution; Health Damage.

\title{
INTRODUÇÃO
}

Nas últimas décadas, com o desenvolvimento das cidades e o aumento da densidade populacional, vários fatores ambientais são questionados diante da sua integridade. Uma dessas questões diz respeito ao ruído ambiental e seu poder de interferir no meio ambiente e na saúde da população.

O ruído ambiental é o resultado da poluição sonora proveniente do exterior, causada por transportes, atividades comerciais, industriais e recreativas.

A poluição sonora ocorre quando, em um determinado ambiente, o som altera a condição normal de audição. Embora ela não se acumule no meio ambiente como os outros tipos de poluição, provoca vários danos ao corpo e a qualidade de vida das pessoas. Segundo a Organização Mundial de Saúde (OMS), a poluição sonora é a segunda maior poluição que afeta a população mundial, ficando atrás apenas da poluição do $\operatorname{ar}^{(1)}$.

O ruído é o que mais colabora para a existência da poluição sonora, sendo definido como um som não desejado que pode afetar de forma negativa a saúde e o bem-estar de indivíduos ou populações ${ }^{(2)}$. Estes ruídos provocam efeitos negativos para o sistema auditivo das pessoas, além de provocar alterações comportamentais e orgânicas. 
Além de problemas de saúde, tais como perda de audição, aumento da pressão arterial, perturbação do sono e queda de desempenho, a elevada exposição ao ruído impacta econômica e financeiramente a vida das pessoas e de organizações ${ }^{(3)}$.

$O$ ruído permeia as atividades humanas durante todo o dia e vem sendo apontado como uma das causas determinantes de deterioração da qualidade de vida. Devido ao tempo de exposição aos ruídos, trabalhadores em canteiros de obras, vendedores ambulantes e guardas de trânsito são alguns dos grupos mais afetados pela alta intensidade do nível de pressão sonora e estão, portanto, mais propensos a sofrerem danos relativos à sua saúde física e mental(4).

Os altos níveis de ruído urbano têm se transformado em uma das formas de poluição de maior preocupação. Os valores registrados acusam níveis de desconforto tão altos que a poluição sonora urbana passou a ser considerada pela OMS como a forma de poluição que atinge o maior número de pessoas. Assim, desde o congresso mundial sobre poluição sonora em 1989, na Suécia, o assunto passou a ser considerado como questão de saúde pública. Entretanto, a preocupação com os níveis de ruído ambiental já existia desde 1981, pois no Congresso Mundial de Acústica, na Austrália, as cidades de São Paulo e Rio de Janeiro passaram a ser consideradas as de maiores níveis de ruído do mundo. Nas cidades médias brasileiras, onde a qualidade de vida ainda é preservada, o ruído já tem apresentado níveis preocupantes, fazendo com que várias delas possuam leis que disciplinem a emissão de sons urbanos ${ }^{(5)}$.

A maioria das pessoas, sobretudo quem trabalha e/ou habita nas cidades, vive um dia-a-dia agitado, sujeito a altos níveis de ruído, na maior parte dos casos muito superiores ao recomendado. Estas fontes de ruído estão ligadas tanto ao progresso tecnológico como aos hábitos de vida e lazer modernos. Para além do barulho que o próprio homem gera, sobretudo a partir de televisões, aparelhos de áudio e outros eletrodomésticos, o ambiente que o rodeia é, do ponto de vista sonoro, cada vez mais agressivo. Automóveis, comboios, aviões e veículos motorizados de duas rodas e até as obras que proliferam um pouco por todo o lado constituem, sem dúvida, as fontes de ruído que mais atormentam ${ }^{(2)}$.

As cidades europeias acima de 250 mil habitantes pela Diretiva 49/EC de 2002 do Parlamento Europeu são obrigadas a desenvolver mapas acústicos para controle da propagação da energia sonora, visando preservar a qualidade ambiental. No Brasil, apesar de existirem alguns dispositivos legais que determinam limites de níveis de ruídos aceitáveis para o controle de poluição sonora, podendo citar as normas NBR 10.151/2019 e NBR 10.152/2017, as resoluções CONAMA $n^{0} .01$ de 1990 e CONAMA $\mathrm{n}^{0}$. 02 de 1990, além de leis estaduais e municipais, o controle acústico não é amplamente difundido. Apesar da Lei Brasileira de Crimes Ambientais $n^{\circ}$. 9.605/98 abordar em seu artigo 54 a poluição sonora como crime, constatam-se nos grandes centros urbanos níveis de ruído acima dos valores recomendados ${ }^{(6-11)}$.

A falta de informações sobre os níveis de ruído e o conhecimento sobre a localização dos pontos críticos gera dificuldade para o setor público interferir e solucionar os problemas, podendo expor a população à poluição sonora. Assim, conhecendo os locais de altos níveis de ruídos vinculados ao planejamento urbano 
melhoraria a qualidade de vida pela redução dos impactos ambientais provocados pelos altos níveis sonoros ${ }^{(12)}$.

Deve ser observado que proteger a saúde da população é o principal objetivo de todos os esforços públicos para controlar a exposição ao ruído do indivíduo ou da comunidade.

A concepção dos espaços urbanos precisa ser gerenciada cuidadosamente a fim de possibilitar a criação de ambientes sonoros agradáveis, capazes de nos proteger dos ruídos indesejáveis, que geram incômodos e acarretam danos à saúde.

No município de Uberaba, Estado de Minas Gerais, existe em torno de 330.000 habitantes e possui diversas fontes de poluição sonora, principalmente em relação à movimentação de veículos automotores e outras fontes de poluição no centro da cidade, como ambulantes. Desta forma é necessária uma avaliação de acústica ambiental da região central da cidade para verificar se os níveis de ruídos estão dentro dos padrões estabelecidos pela legislação e normas brasileiras e o quanto estes ruídos estão afetando a saúde e o bem-estar da população.

\section{METODOLOGIA}

O estudo foi realizado por meio de medições de nível de pressão sonora em 5 locais selecionados da região central de Uberaba - MG. A metodologia de medição seguiu as recomendações da norma brasileira NBR 10151/2019 e se dividiu em 6 etapas.

$1^{\text {a }}$ Etapa: Delimitação dos locais de medição

As medições foram realizadas na região central de Uberaba e nas principais vias de acesso ao centro da cidade. A partir de uma análise subjetiva de percepção sonora pelos próprios autores, foram escolhidas 5 zonas do centro urbano (Figura 1), para realizar as medições. Também foi considerado para escolhas destas áreas o grande volume no tráfego de veículos automotores, a participação nas rotas itinerárias de várias linhas do transporte público coletivo, a presença de grandes pontos comerciais e prédios públicos e alta taxa de circulação de pedestres. 
Figura 1 - Zonas de estudos na região central de Uberaba - MG

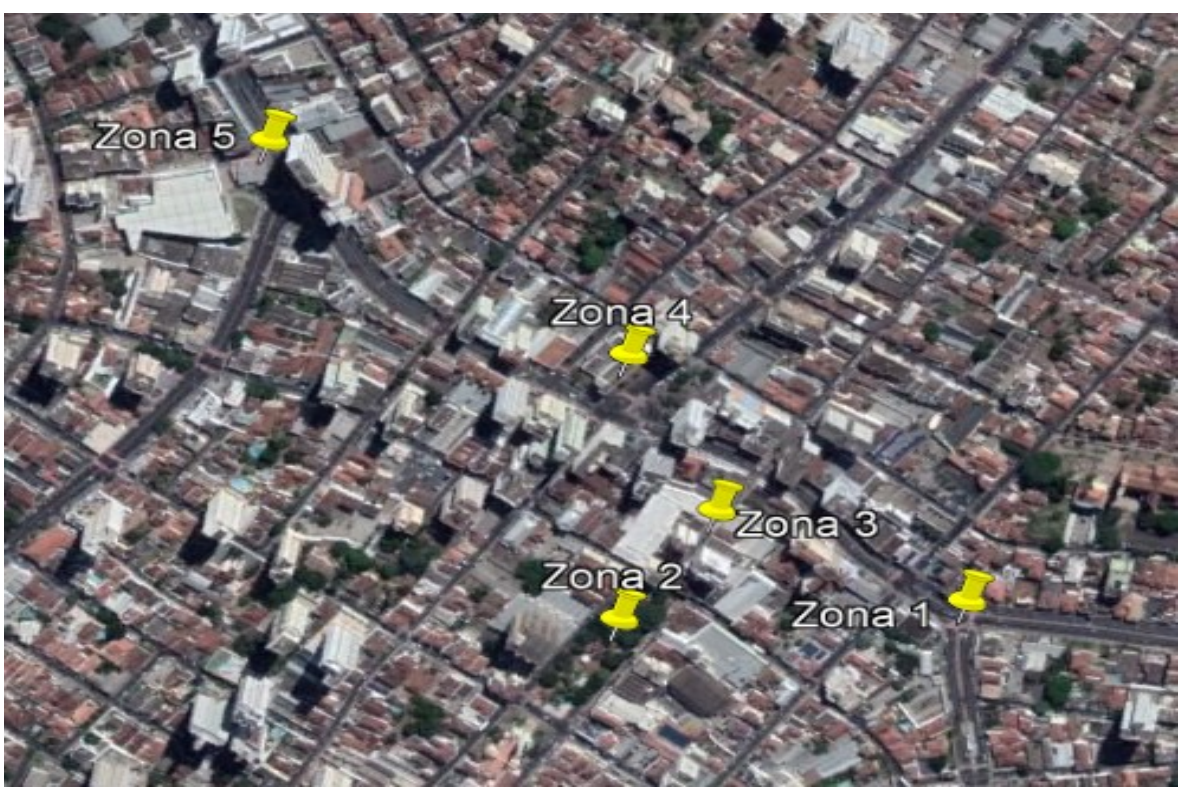

Fonte: Google Earth(13)

A zona 1 e zona 5 corresponde a um cruzamento de duas avenidas que possuem tráfego intenso de veículos, ocorrendo engarrafamento nos períodos de pico observados entre os horários de 17:00 e 18:30.

A zona 2 diz respeito a praça central da cidade, que em seu entorno tem vários estabelecimentos comerciais, como lanchonetes, óticas, lojas de roupas populares e lojas de eletroeletrônicos. Muitos destes estabelecimentos fazem propagandas sonoras com caixas de som na sua porta. Além disso, a praça possui um ponto de ônibus com frequência de passagem de coletivos a cada 5 minutos e um grande número de pessoas e ambulantes circulando.

A zona 3 refere-se a um calçadão próximo à praça central que possui a presença de vários ambulantes, estabelecimentos comerciais com propagandas sonoras e circulação de pedestres.

Já na zona 4 está localizado o edifício central dos correios e dá acesso à Avenida Leopoldino de Oliveira, avenida considerada pela percepção subjetiva a de maior fluxo de veículos automotores. Sendo assim foram definidas:

-Zona 1 - Cruzamento da Avenida Guilherme Ferreira com a Avenida Leopoldino de Oliveira;

-Zona 2 - Praça Rui Barbosa;

-Zona 3 - Calçadão;

-Zona 4 - Edifício Central dos Correios da Avenida Leopoldino de Oliveira; 
-Zona 4 - Cruzamento da Avenida Santos Dumont com a Avenida Leopoldino de Oliveira.

\section{$2^{a}$ Etapa: Medição de Nível de Pressão Sonora}

Para a medição de ruídos foi utilizado um aparelho de medição de Nível de Pressão Sonora (NPS), conhecido popularmente como "decibelímetro". O modelo utilizado foi o decibelímetro digital AK820 da marca AKSO ${ }^{\circledR}$.

As medições sonoras foram realizadas na escala decibel $(\mathrm{dB})$ ponderados na curva de calibração $A$, que é a calibração mais semelhante ao ouvido humano. Foram seguidos os critérios de medição definidos pela NBR 10.151/2019 com altura de medição de 1,40 m do chão e distância de $2 \mathrm{~m}$ de qualquer superfície.

Foram realizadas medições de ruído de fundo, que consiste do ruído ambiente, ou seja, sem movimentação e medições de ruído de fonte + fundo.

As medições de ruído de fundo foram realizadas nos finais de semana nos quais os estabelecimentos comerciais estavam fechados e tem um menor fluxo de pessoas e veículos automotores.

As medições de ruído de fonte + fundo foram feitas em dia e horário de maior movimentação e maior intensidade acústica.

Tanto as medições de ruído de fundo como as medições de ruído de fonte + fundo foram realizadas no mesmo horário e nos mesmos pontos, porém em dias diferentes. Para cada uma das 5 zonas estabelecidas de medição selecionou-se 4 pontos, sendo que para cada ponto realizaram-se 10 leituras de NPS no intervalo de 1 minuto cada leitura, totalizando 40 medições de ruído de fundo e 40 medições de ruído de fonte + fundo para cada zona.

\section{$3^{a}$ Etapa: Tratamento de dados}

Os valores de NPS foram tratados para obtenção da intensidade acústica e a partir dela, o Nível de Pressão Sonora Equivalente (NPSeq), que corresponde à média dos valores medidos e representa o nível de pressão sonora do local avaliado, foi comparado com os limites aceitáveis pelas normas para verificação de insalubridade local.

Calculou-se a intensidade acústica para cada uma das medidas dos quatro pontos de medição de cada zona por meio da Equação 1.

$$
\mathrm{NPS}=10 \cdot \log \left(\frac{\mathrm{I}}{\mathrm{I}_{\text {ref }}}\right)
$$


Onde:

I = Intensidade acústica

Iref $=$ Intensidade acústica de referência, valor de $10^{-12} \mathrm{w} / \mathrm{m}^{2}$ que é equivalente ao ar, que foi o meio de propagação analisado.

Utilizando as equações 2 e 3, calculou-se, também, a intensidade acústica média (Imédio) e o NPSeq para cada ponto, no qual foram realizadas as medições.

$$
\begin{gathered}
\mathrm{I}_{\text {médio }}=\frac{1}{\mathrm{~T}} \cdot \int \mathrm{I}(\mathrm{t}) \cdot \mathrm{dt}=\frac{\sum_{\mathrm{i}=0}^{9} \mathrm{Ai}}{9} \\
\mathrm{NPS}_{\text {eq }}=10 \cdot \log \left(\frac{\mathrm{I}_{\text {médio }}}{\mathrm{I}_{\text {ref }}}\right)
\end{gathered}
$$

O valor médio do nível de pressão sonora para o ruído de fundo foi definido através da média aritmética dos NPSeq de fundo de cada ponto. O mesmo procedimento foi aplicado para o valor médio de pressão sonora para o ruído de fonte + fundo.

4ª Etapa: Análise dos resultados

Os resultados foram comparados com as normas e leis que determinam o conforto acústico necessário aos ambientes externos e que definem o ruído de fundo permitido. Foram utilizadas a NBR 10.151/2019 (7), Lei 10.100 do Estado de Minas Gerais ${ }^{(14)}$, que equivale ao mesmo Limite estabelecido pela Resolução CONAMA n. 01 de $1990{ }^{\left({ }^{(9)}\right.}$ e os critérios estabelecidos pela OMS.

5a Etapa: Proposição de solução

Baseado nos resultados indicou-se algumas medidas mitigadoras que podem ser aplicadas para atenuação dos níveis sonoros da região central de Uberaba.

\section{RESULTADOS}

Devido a quantidade de dados, 40 medições de ruído de fundo e 40 medições de ruído de fonte + fundo para cada zona, a zona 1 será mostrada como exemplo dos 
resultados alcançados e para as demais zonas só será apresentando o resultado de Nível de Pressão Sonora de cada ponto de medição e o Nível de Pressão Sonora Equivalente (NPSeq), que corresponde a intensidade sonora do local avaliado.

A Tabela 1 mostra os resultados de nível de pressão sonora de ruído de fundo e ruído de fonte + fundo medidos nos 4 pontos selecionados da zona 1 e com a aplicação da Equação 1 obteve parâmetros para utilização das Equações 2 e 3 conseguindo os valores de intensidade sonora média e NPSeq.

Tabela 1 - Valores medidos em cada ponto da Zona 1

\begin{tabular}{|c|c|c|c|c|c|c|c|c|}
\hline \multicolumn{9}{|c|}{ Pontos Medidos (dB) } \\
\hline \multirow{2}{*}{$\begin{array}{c}\text { Temp } \\
\text { o } \\
\text { Min }\end{array}$} & \multicolumn{2}{|c|}{ Ponto 1} & \multicolumn{2}{|c|}{ Ponto 2} & \multicolumn{2}{|c|}{ Ponto 3} & \multicolumn{2}{|c|}{ Ponto 4} \\
\hline & $\begin{array}{c}\text { Fund } \\
0\end{array}$ & $\begin{array}{c}\text { Fonte + } \\
\text { Fundo }\end{array}$ & Fundo & $\begin{array}{c}\text { Fonte + } \\
\text { Fundo }\end{array}$ & Fundo & $\begin{array}{c}\text { Fonte + } \\
\text { Fundo }\end{array}$ & Fundo & $\begin{array}{c}\text { Fonte + } \\
\text { Fundo }\end{array}$ \\
\hline 1 & 63,8 & 67,6 & 68,0 & 70,6 & 64,8 & 75,0 & 74,8 & 76,2 \\
\hline 2 & 61,2 & 70,9 & 69,4 & 77,2 & 69,6 & 72,6 & 65,2 & 65,6 \\
\hline 3 & 66,7 & 75,8 & 68,2 & 77,6 & 69,4 & 71,5 & 70,1 & 59,0 \\
\hline 4 & 77,2 & 75,1 & 63,2 & 73,8 & 77,3 & 69,9 & 73,2 & 79,6 \\
\hline 5 & 69,5 & 71,5 & 75,7 & 74,0 & 67,3 & 73,5 & 72,3 & 73,7 \\
\hline 6 & 63,1 & 72,9 & 73,4 & 63,8 & 73,4 & 74,5 & 59,1 & 70,6 \\
\hline 7 & 70,5 & 69,2 & 55,8 & 68,0 & 66,2 & 65,9 & 60,5 & 66,0 \\
\hline 8 & 64,4 & 73,8 & 67,8 & 66,1 & 73,1 & 75,3 & 71,1 & 61,6 \\
\hline 9 & 69,6 & 67,3 & 66,5 & 75,5 & 66,8 & 68,4 & 65,2 & 70,8 \\
\hline 10 & 63,4 & 82,3 & 75,2 & 80,0 & 68,1 & 70,8 & 76,0 & 63,2 \\
\hline $\begin{array}{c}\text { Imédi } \\
0\end{array}$ & $\begin{array}{c}1,1 \times 1 \\
0^{-5} \\
\end{array}$ & $2,7 \times 10^{-5}$ & $\begin{array}{c}1,2 x \\
10^{-5} \\
\end{array}$ & $2,9 \times 10^{-5}$ & $\begin{array}{c}1,5 x \\
10^{-5} \\
\end{array}$ & $1,8 \times 10^{-5}$ & $\begin{array}{c}1,2 \times \\
10^{-5} \\
\end{array}$ & $1,9 \times 10^{-5}$ \\
\hline $\begin{array}{c}\text { NPSe } \\
q\end{array}$ & 70,2 & 74,3 & 70,8 & 74,7 & 71,7 & 72,5 & 70,7 & 72,8 \\
\hline
\end{tabular}

Pelos resultados da Tabela 1 pode-se notar pequena diferença entre os valores de ruído de fundo e ruído de fonte + fundo, evidenciando que mesmo nos finais de semana o nível de ruído da Zona 1 é elevado. Este ruído é advindo principalmente pela movimentação de veículos automotores.

A Figura 2 apresenta a variação de nível de pressão sonora de fonte + fundo de cada ponto de medição dos locais de estudo. 
Figura 2 - Variação do nível de pressão sonora dos locais de medição

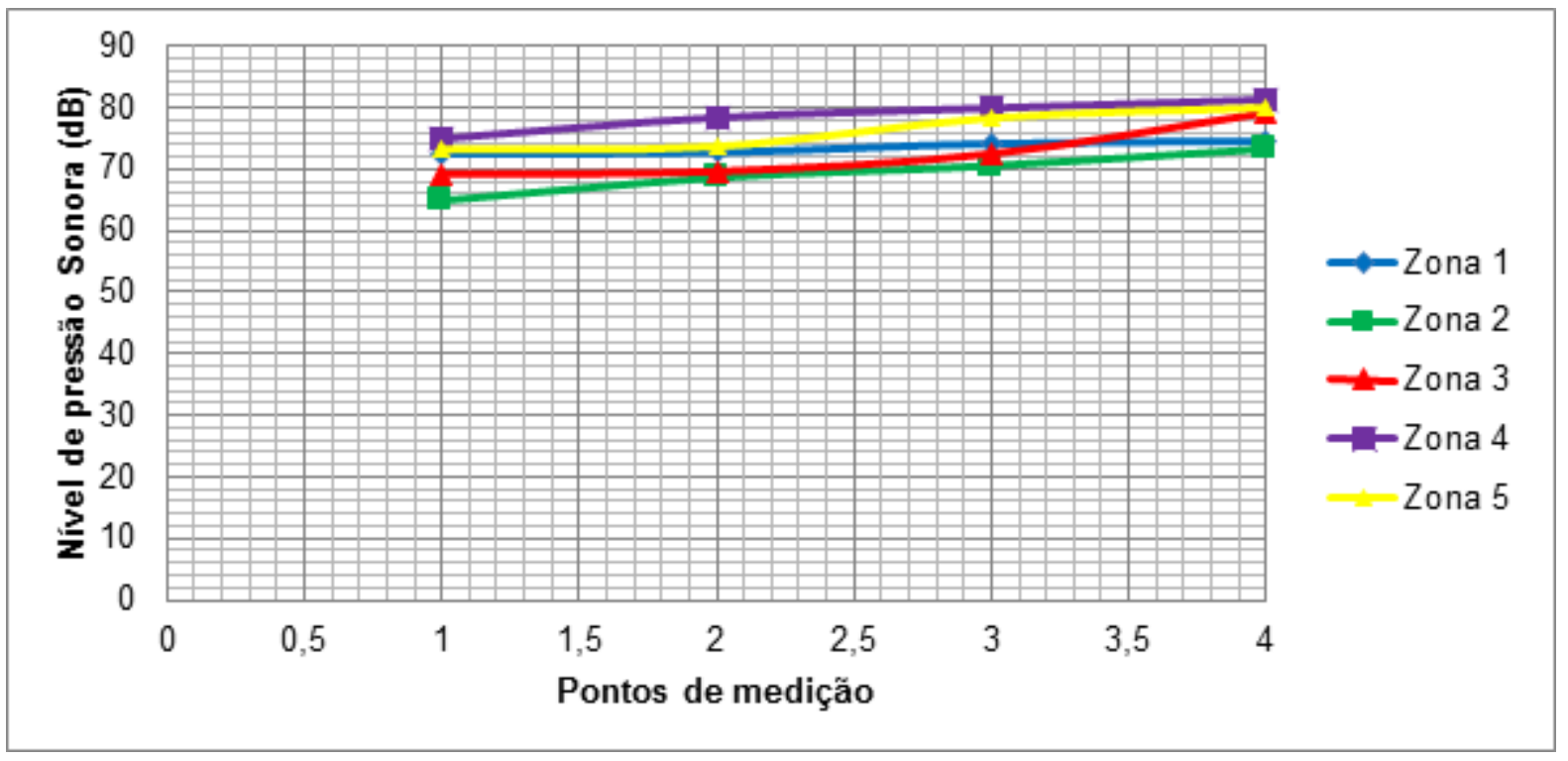

É observada na Figura 2 que a zona 4 apresenta o maior nível de pressão sonora em todos os 4 pontos medidos em comparação com as demais zonas. Isso pode ser justificado devido ser o local de maior fluxo de veículos automotores.

Há pouca variação sonora entre os pontos medidos de cada zona, com um aumento de aproximadamente $5 \mathrm{~dB}$ entre os pontos. A maior variação sonora é observada na zona 3 , no qual no Ponto 1 o valor fica em torno de $69 \mathrm{~dB}$ e no ponto 4 aproximadamente $80 \mathrm{~dB}$. A zona 2 apresenta também uma variação de nível de pressão sonora em torno de $10 \mathrm{~dB}$.

A variação de NPS entre os pontos da zona 2 pode ser explicada devido ao ponto de ônibus na praça que possui passagem de coletivo a cada 5 minutos. Durante a medição do ponto 4 haviam dois ônibus ligados parados esperando a entrada de passageiros.

Já a diferença sonora entre os pontos da zona 3 pode ser esclarecida pelo fluxo de pedestres conversando e ambulantes com elevado tom de voz no oferecimento de produtos.

Para obtenção do NPSeq de fundo e NPSeq de fonte + fundo do local avaliado tirou-se a média dos 4 valores de NPSeq mostrados na Tabela 1. Com isso tem-se para ruído de fundo um NPSeq de 70,9 dB e para ruído de fonte + fundo 73,6 dB. Estes constituem o valor representativo da medição deste local.

O mesmo procedimento de cálculo também foi realizado para as demais zonas, obtendo assim o NPSeq para cada local de medição efetuada. A Tabela 2 apresenta os resultados de NPSeq de fundo e fonte + fundo para cada zona estudada. 
Tabela 2 - NPSeq de ruído de fundo e ruído de fonte+fundo de cada local de medição

\begin{tabular}{c|c|c|c|c|c}
\hline Medições & Zona 1 & Zona 2 & Zona 3 & Zona 4 & Zona 5 \\
\hline Ruído de fundo & 70,9 & 63,9 & 62,7 & 67,4 & 65,4 \\
\hline Ruído de fonte+fundo & 73,6 & 69,3 & 72,5 & 78,7 & 76,3 \\
\hline
\end{tabular}

Podem ser observados pela Tabela 2 elevados valores de ruído de fundo. $O$ alto valor obtido na Zona 2 pode ser justificado pela praça ser utilizada aos finais de semana para recreação. No dia da coleta de dados as perturbações foram causadas por um grupo de pessoas que fazia práticas esportivas de skate.

De acordo com a NBR 10.151 o limite de nível sonoro para áreas mistas com predominância de atividades culturais, lazer e turismo é de $65 \mathrm{~dB}^{(7)}$. Sendo assim, analisando o ruído ambiente, nos finais de semana a zona 2 se constitui de um ambiente salubre acusticamente.

Os outros locais avaliados se enquadram de acordo com a NBR 10.151 como áreas mistas com predominância de atividades comerciais e/ou administrativa que estabelece um valor limite de $60 \mathrm{~dB}$. Dessa forma todas as zonas são consideradas insalubres. São notadas pela Tabela 2 pequenas diferenças entre ruído de fundo e ruído de fonte + fundo. A maior diferença é de 11,4 dB, observada na Zona 4.

A Lei 10.100 considera que para as cidades do Estado de Minas Gerais os ruídos prejudiciais à saúde, segurança e sossego público devem alcançar a diferença de $10 \mathrm{~dB}$ entre ruído de fundo e ruído de fonte + fundo. A lei municipal de Uberaba segue o estabelecido por ela ${ }^{(14)}$. Sendo assim, de acordo com esta lei, as Zonas 3, 4 e 5 são consideradas deletérias. Na zona 3 o maior ruído foi notado pela presença de ambulantes. Já nas Zonas 4 e 5 há predominância de movimentação de veículos automotores. A Lei 10.100 ainda diz que o ambiente está insalubre se independente do ruído de fundo, o local analisado atinja um nível de pressão sonora de $70 \mathrm{~dB}$ durante o dia, logo considerando esta prescrição, apenas a zona 2 seria considerada um ambiente confortável acusticamente.

A OMS estipulou que o limiar de incômodo para o ruído é na faixa de $50 \mathrm{~dB}$. No período noturno, os níveis sonoros devem situar-se entre os 5 e $10 \mathrm{~dB}$ abaixo dos valores diurnos, para garantir um ambiente sonoro equilibrado ${ }^{(1)}$.

Observando os valores de NPS da Tabela 2 todos os valores tanto de ruído de fundo, quanto de ruído de fonte + fundo estão acima do estabelecido para conforto acústico, segundo a OMS.

O Quadro 1 mostra os efeitos dos ruídos na saúde humana de acordo com o nível de pressão sonora. 
Michele Cristina Rufino Barbosa; Guilherme Wolf Gothelf; Lucas Araújo Carvalho; Mateus Scandar Prata

Quadro 1 - Impactos dos ruídos na saúde humana

\begin{tabular}{|c|c|l|}
\hline $\begin{array}{c}\text { Intensidade } \\
\text { Sonora }\end{array}$ & Reação & \multicolumn{1}{c|}{ Efeitos Negativos } \\
\hline Até $50 \mathrm{~dB}$ & $\begin{array}{c}\text { Confortável } \\
\text { (limite da OMS) }\end{array}$ & \multicolumn{1}{c|}{ Nenhum } \\
\hline Acima de 50dB & \multicolumn{2}{|c|}{ Organismo humano começa a sofrer impactos do ruído } \\
\hline 55 a $65 \mathrm{~dB}$ & $\begin{array}{c}\text { Pessoa fica em estado } \\
\text { de alerta, não relaxa }\end{array}$ & $\begin{array}{l}\text { Diminui poder de concentração } \\
\text { Prejudica a produtividade intelectual } \\
\text { e no trabalho }\end{array}$ \\
\hline 65 a $70 \mathrm{~dB}$ & $\begin{array}{c}\text { Organismo reage para } \\
\text { tentar adaptar-se ao } \\
\text { Ambiente }\end{array}$ & $\begin{array}{l}\text { Diminuição da resistência imunológica } \\
\text { Aumenta concentração de colesterol } \\
\text { no sangue }\end{array}$ \\
\hline Acima de $70 \mathrm{~dB}$ & $\begin{array}{c}\text { Organismo fica sujeito } \\
\text { a stresse degenerativo, } \\
\text { além de abalar a saúde } \\
\text { Mental }\end{array}$ & $\begin{array}{l}\text { Provoca doenças cardiovasculares, } \\
\text { como a hipertensão e paragens } \\
\text { cardíacas. } \\
\text { Amentam riscos de infecções e } \\
\text { outras doenças sérias. }\end{array}$ \\
\hline
\end{tabular}

Fonte: Vianna $(2014)^{(15)}$

Comparando o NPSeq obtido em cada zona de medição com os valores estabelecidos na Tabela 2 são verificados impactos negativos dos ruídos na saúde humana.

\section{DISCUSSÃO}

No estudo foi constatada a poluição sonora nas áreas centrais da cidade de Uberaba podendo afetar a saúde e o bem-estar da população que ali frequentam.

A poluição sonora no meio urbano tem sido uma constante em muitas cidades brasileiras sendo apontada pela OMS como um dos problemas mais comuns das cidades afetando a qualidade de vida e degradando o meio ambiente.

Assim como no presente trabalho, a avaliação do ruído em áreas urbanas também foi objeto de estudo em pesquisas semelhantes, tais como Mardones (2009); Silva (2009); Mendonça et al. (2013); Brasileiro (2017); Magioli e Torres (2018), sendo que em todos os estudos, os ruídos estavam acima dos níveis aceitáveis estabelecidos pela legislação, observando-se a presença da poluição sonora nas cidades $^{(16-20)}$.

Levando em consideração os limites aceitáveis de ruídos estabelecidos por Lei Municipais, Calixto (2002) estudou o problema do ruído urbano gerado pelo tráfego de veículos nas rodovias BR-116 e BR-277, que se transformaram em grandes 
avenidas na cidade de Curitiba - PR e constatou que os níveis do ruído de tráfego nas "rodovias-grandes avenidas" estudadas são superiores aos permitidos pela legislação municipal. No estudo de Andrade (2012), também desenvolvido na cidade de Curitiba - PR foram medidos níveis de ruídos de 55 pontos da região central da cidade e todos os pontos medidos apresentaram níveis de ruído superiores ao valor limite estabelecido pela lei municipal, na qual define que no período diurno $(07 \mathrm{~h} 01 \mathrm{~min}$ até $19 \mathrm{~h} 00 \mathrm{~min}$ ) o limite de pressão sonora é de $65 \mathrm{~dB}$. Isso mostra a poluição sonora na cidade de Curitiba não é recente, desde 2002 o município já sofre com problemas de níveis sonoros elevados ${ }^{(21-22)}$.

Os resultados confirmam a necessidade do controle e planejamento acústico da cidade de Uberaba. A análise do ruído no meio urbano deve ser considerada juntamente com o planejamento urbano. Seguindo o exemplo das cidades europeias para prevenir impactos sonoros e diagnosticar áreas com conflitos acústicos já existentes para tomar medidas corretivas, o mapeamento sonoro é uma ferramenta de grande auxílio para planejamento urbano.

O mapa sonoro de uma cidade pode ser utilizado para várias finalidades: identificar as principais fontes de ruído urbano, demonstrar a propagação de ruído no meio ambiente, servir de base para uma política pública de controle de ruído considerando o custo versus benefício das ações, ajudar a desenvolver ações de punição a nível regional e nacional para reduzir a emissão de energia sonora, além de garantir a existência de áreas tranquilas próximas aos centros urbanos, monitorar o processo de redução de ruído durante a implantação de políticas públicas, monitorar as alterações no padrão acústico das cidades e servir de base para estudos do efeito do ruído na população em geral. O mapa acústico também pode fornecer informações detalhadas sobre o impacto do ruído na população, sendo uma ferramenta científica para compreender o fenômeno de propagação de ruído no meio ambiente ${ }^{(23)}$.

O mapa acústico da cidade mostrando os locais de maior nível de pressão sonora serve para orientar as políticas públicas relacionadas com o sistema de transportes e o uso do solo, de modo a racionalizar os deslocamentos e reduzir os impactos sobre o meio ambiente e a qualidade de vida.

O maior problema evidenciado no estudo foi em relação aos ruídos emitidos por veículos automotores. Uma medida de atenuação sonora e controle de ruídos seria a realização de campanhas que conscientizem sobre o impacto negativo do ruído na saúde e incentivem à população a reduzir o uso de veículos automotores estimulando a utilização de transportes coletivos. Deve-se incentivar também a utilização de modos não motorizados através da expansão da malha ciclo viária da cidade. Outra medida seria o controle do número de veículos pesados e médios, pois estes são os maiores emissores de ruído ao meio ambiente. A velocidade dos veículos também deve ser controlada por meio de fiscalização.

Outra forma de atenuação de ruídos seriam campanhas educativas incentivando a correta manutenção dos veículos. Em relação aos veículos de transporte coletivo a prefeitura deve fazer um controle rigoroso destas frotas verificando os níveis de emissão sonora, exigindo, sua manutenção periódica ou a sua substituição por veículos mais novos e silenciosos. 
A reflexão no solo, por exemplo, pode resultar em atenuação ou amplificação do nível sonoro, em função do tipo de material. Dessa forma, podem-se obter reduções de 20 a $30 \mathrm{~dB}$ ou amplificações de até $6 \mathrm{~dB}$. A propagação sobre solos acusticamente "duros", como asfalto e terra batida resulta em amplificações médias de aproximadamente $3 \mathrm{~dB}$, em relação ao som direto. Já a propagação sobre solos acusticamente "macios", como vegetação e solo arado resulta em significativa atenuação do nível sonoro (2). Portanto, a simples substituição do material de superfície das vias por asfalto emborrachado ou asfalto poroso poderia reduzir o ruído de rolagem em 8 a $10 \mathrm{~dB}^{(24)}$. Associada a esta medida, a adoção de barreiras acústicas naturais, com arbustos, por exemplo, também poderia reduzir os níveis de ruído entre 10 e $20 \mathrm{~dB}$, se as medidas forem utilizadas de forma conjugada(25). Sendo assim, o ideal seria a substituição do asfalto convencional das vias da região central de Uberaba por asfalto poroso.

Outra iniciativa, mais especificamente voltada para os ambulantes e os trabalhadores de estabelecimentos comerciais constituiria no uso de protetores auriculares. Especificamente para zona 2 uma medida de atenuação seria a introdução de vegetação em torno praça e também a colocação de barreiras acústicas no ponto de ônibus.

\section{CONCLUSÃO}

Por meio das medições, comprovou-se que a região central de Uberaba é acusticamente insalubre. As medições em todos os pontos excederam os limites estabelecidos pelas normas e a legislação.

O resultado já era esperado, pois nas áreas centrais das cidades, a quantidade de pessoas que se expõe diariamente ao ruído do ambiente externo é grande, pela própria característica dos centros urbanos de concentrarem um alto número de atividades socioeconômicas. Além dos pedestres, podem-se citar ainda os comerciantes, policiais e ambulantes que não utilizam qualquer tipo de proteção auditiva e atuam por períodos prolongados nestes locais.

O ruído deve ser encarado com mais seriedade, tanto pelos trabalhadores como pelos órgãos públicos, sendo necessária a realização de campanhas que conscientizem sobre o impacto negativo do ruído na saúde humana. A educação é imprescindível para a disseminação de bons hábitos quanto ao controle da qualidade ambiental, em particular no que diz respeito à poluição sonora. $\mathrm{O}$ município deve fiscalizar e colocar em prática as leis em vigor sobre os limites de ruídos e investir em planejamento urbano.

A poluição sonora deve fazer parte do planejamento urbano das cidades para garantir à saúde da população. Políticas públicas de mobilidade urbana, redução de veículos automotores nas vias urbanas, incentivo à utilização de transportes coletivos públicos e ao uso de veículos não motorizados devem fazer parte deste planejamento. 
O levantamento dos locais críticos através do mapeamento sonoro da cidade também possibilitará o controle dos ruídos.

No Brasil não há preocupações com o ambiente sonoro no meio urbano, havendo grande necessidade de serem ampliados os estudos sobre o tema. São necessárias medidas preventivas e ações corretivas no planejamento urbano, bem como a divulgação para a comunidade sobre o ambiente sonoro a que está exposta, para que haja também uma preocupação da sociedade em geral acerca da problemática.

Os níveis excessivos de ruídos causam deterioração na qualidade de vida, e na relação entre as pessoas, sobretudo nos centros urbanos. Assim, é imprescindível que se iniciem em todos os municípios brasileiros ações de planejamento de desenvolvimento urbano.

\section{REFERÊNCIAS}

1- World Health Organization [homepage na internet]. Constitution of the World Health Organization [acesso em 15 jun 2019]. Disponível em: https://www.who.int/governance/eb/who_constitution_en.pdf.

2- Bistafa SR. Acústica Aplicada ao Controle de Ruído. 2.ed. São Paulo: Editora Edgard Blucher; 2011.

3- Anais do XIII Encontro Nacional e IX Encontro Latino-americano de Conforto no Ambiente Construído; 15 - 17 out 2015; Campinas (SP): PUC Campinas; 2015.

4- Brito LAPF. A utilização de mapas acústicos como ferramenta de identificação do excesso de ruído em áreas urbanas. Rev Eng Sanitária e Ambiental. 2017; 22 (6): 163-169.

5- Souza Filho JJ. Avaliação do ruído urbano na cidade de Campo Grande/MS [dissertação]. Campo Grande (MS): Universidade Federal de Mato Grosso do Sul; 2012.

6- Parlamento Europeu [homepage na internet]. Directiva 2002/49/EC: Assessment and management of environmental noise [acesso em 04 nov 2018]. Disponivel em: https://eurlex.europa.eu/legalcontent/PT/\%20TXT/ ?uri=celex\%3A32002L0049.

7- Associação Brasileira de Normas Técnicas. NBR 10.151: Acústica - Avaliação do ruído em áreas habitadas visando o conforto da comunidade - Procedimento. ABNT. 31 maio 2019.

8- Associação Brasileira de Normas Técnicas. NBR 10.152: Níveis de ruído para conforto acústico. ABNT. 24 nov 2017. 
9- Brasil. Resolução CONAMA n¹, de 08 de março de 1990. Dispõe sobre critérios de padrões de emissão de ruídos decorrentes de quaisquer atividades industriais, comerciais, sociais ou recreativas, inclusive as de propaganda política. Diário Oficial da União. 2 abr 1990.

10- Brasil. Resolução CONAMA n², de 08 de março de 1990. Dispõe sobre o Programa Nacional de Educação e Controle da Poluição Sonora. Diário Oficial da União. 2 abr 1990.

11- Brasil. Lei $n^{\circ} 9.605$, de 12 de fevereiro de 1998. Dispõe sobre as sanções penais e administrativas derivadas de condutas e atividades lesivas ao meio ambiente, e dá outras providências. Diário Oficial da União. 13 fev 1998.

12- Suarez E, Barros JL. Traffic noise mapping of the city of Santiago de Chile. Sci Total Environment. 2014; 466 (1):539-546.

13- Google Earth [homepage na internet]. Localização no mapa da área central de Uberaba - MG [acesso em 10 nov 2018]. Disponível em: https://www.google.com.br/intl/pt-BR/earth/

14- Minas Gerais. Lei 10.100, de 17 de janeiro de 1990. Dispõe sobre a proteção contra a poluição sonora no Estado de Minas Gerais. Diário Executivo de Minas Gerais. 18 jan 1990.

15- Vianna KMP. Poluição Sonora no Município de São Paulo: Avaliação do ruído e o impacto da exposição na saúde da população [tese]. São Paulo (SP): Universidade de São Paulo; 2014.

16- Mardones MDM. Mapeamento dos níveis de ruído em Copacabana, Rio de Janeiro, através de simulação computacional [dissertação]. Rio de Janeiro (RJ): Universidade Federal do Rio de Janeiro; 2009.

17- Silva ASM. Acústica ambiental: análise de ruído urbano [dissertação]. Porto (PT): Universidade do Porto; 2009.

18- Mendonça ABD, Suriano MT, Souza LCL, Viviani E. Classes de quadras urbanas determinadas pelos níveis de ruídos. Rev Bras de Gestão Urbana. 2013; 5(2): 63-77.

19- Brasileiro TM. Mapeamento Sonoro: Estudo do Ruído Urbano no Bairro Castelo Branco, em João Pessoa/PB [dissertação]. Natal (RN): Universidade Federal do Rio Grande do Norte; 2017.

20- Magioli FB, Torres JCB. Influência das transformações urbanas no conforto acústico: estudo-piloto da cidade universitária da UFRJ. Rev Bras de Gestão Urbana. 2018; 10(2): 400-413. 
21- Calixto A. O ruído gerado pelo tráfego de veículos em "rodovias-grandes avenidas" situadas dentro do perímetro urbano de Curitiba: analisado sob parâmetros acústicos objetivos e seu impacto ambiental [dissertação]. Curitiba (PN): Universidade Federal do Paraná; 2002.

22- Andrade FC. Elaboração de um Mapa Acústico para a Região Central de Curitiba e Principais Vias de Acesso ao Centro da Cidade [dissertação]. Curitiba (PN): Universidade Tecnológica Federal do Paraná; 2012.

23- Giunta MB. Análise de modelagem de previsão acústica e mapeamento sonoro para a cidade de São Carlos - SP [dissertação]. São Carlos (SP): Universidade Federal de São Carlos; 2013.

24- Morgan PA, Watts GR. A novel approach to the acoustic characterisation of porous road surfaces. Applied Acoustics. 2003; 64(12): 1171-1186.

25- Anais do $19^{\circ}$ Congresso Brasileiro de Transporte e Trânsito; 8 - 10 out 2013; Brasília (DF): ANTP; 2013.

Artigo recebido em: 20/10/2020

Artigo aprovado em: 13/04/2020

Artigo publicado em: 06/05/2020 